\title{
EEG DATA PROCESSING IN ADHD DIAGNOSIS AND NEUROFEEDBACK
}

\author{
Zoran Šverko* - Saša Vlahinić - Miroslav Vrankić - Ivan Markovinović \\ Department of Automation and Electronics, Faculty of Engineering, University of Rijeka, Vukovarska 58, 51000 Rijeka, \\ Croatia
}

\begin{tabular}{l}
\hline ARTICLE INFO \\
\hline Article history: \\
Received: 4.6 .2019$. \\
Received in revised form: 20.11 .2019$. \\
Accepted: 28.11 .2019$. \\
\hline Keywords: \\
EEG \\
Data processing \\
Neurofeedback \\
\hline DOI: http://doi.org/10.30765/er.40.3.12 \\
\\
\end{tabular}

\section{Introduction}

Attention-deficit hyperactivity disorder (ADHD) is a mental disorder of the neurodevelopmental type [1], [2]. It is characterized by problems with paying attention, excessive activity or difficulty in controlling behavior which is not appropriate for a person's age [3], [4]. When it comes to children, problems with paying attention may result in poor school performance [3]. As of 2015, ADHD is estimated to affect about 51.1 million people globally [5]. Neurofeedback is a type of biofeedback that measures brain waves to produce a signal that can be used as a feedback to teach self-regulation of brain function. Video or sound are commonly used for neurofeedback [6]. In neurofeedback, electroencephalography (EEG) signals of ADHD participants are recorded in real time in order to stimulate participants with sound, video and pictures.

\begin{abstract}
:
In this paper, EEG data processing was conducted in order to define the parameters for neurofeedback. A new survey was conducted based on a brief review of previous research. Two groups of participants were chosen: ADHD (3) and non$A D H D$ (14). The main part of this study includes EEG signal data pre-processing and processing. We have outlined statistical features of observed EEG signals such as mean value, grand-mean value and their ratios. It can be concluded that an increase in grand-mean values of power theta-low beta ratio on $\mathrm{Cz}$ electrode gives confirmation of previous research. The value of alpha-delta power ratio higher than 1 on $\mathrm{C} 3, \mathrm{Cz}, \mathrm{P3}, \mathrm{Pz}, \mathrm{P} 4$ in $\mathrm{ADHD}$ group is proposed as a new approach to classification. Based on these conclusions we will design a neurofeedback protocol as a continuation of this work.
\end{abstract}

EEG is an electrophysiological monitoring method that records electrical activity of the brain [7]. Thus, EEG provides signatures of neural activities [8]. Furthermore, multimodal approach is advised for the cure of ADHD. This approach is a combination of different types of treatments: medication (stimulants), psychoeducation, psychological treatment. As a consequence of personal preferences, some participants or their parents are unwilling to use medication. Because of that reason, neurofeedback has a great opportunity to become a drug-free alternative treatment for ADHD [9]. In order to record the signals, electrodes are commonly placed on the scalp. Figure 1 shows the placement of electrodes. EEG signals can be recorded with a different number of electrodes. In general, one or a few electrodes are used during neurofeedback protocol. We can observe EEG signals as waveforms that are defined by their amplitude, frequency and

\footnotetext{
* Corresponding author. Tel.: +385 51505720

E-mail address: zsverko@ riteh.hr
} 
place of origin. EEG waveforms are usually separated into delta, theta, alpha, beta and gamma waves. Delta waves usually appear in a person's deep sleep, in children and in case of severe brain illnesses. Their frequency lies between 1 to $4 \mathrm{~Hz}$, the amplitude ranges from 60 to $100 \mu \mathrm{V}$ and the place of origin is the cortex. The theta wave frequencies are between 4 and $8 \mathrm{~Hz}$, the amplitude goes up to $70 \mu \mathrm{V}$, and the places of origin are the temporal and the parietal lobe. They appear in all age groups and are caused by stress during examination tests [10], emotional disappointment, frustration and also idling thinking. A frequency of 8 to $13 \mathrm{~Hz}$, amplitude up to $50 \mu \mathrm{V}$ are typical parameters of alpha waves. The place of origin is the occipital lobe. They most often appear in an awake but relaxed state, when participants are unfocused. Beta waves appear during mental activity. Their frequency is between 13 and $30 \mathrm{~Hz}$ and their amplitude amounts up to $20 \mu \mathrm{V}$. The places of origin are the frontal and the parietal lobe. During the EEG signal recording, after participant's eyes are closed and the participant opens the eyes, alpha waves are blocked and beta waves appear. Gamma waves appear during high-level information processing. Their frequency is from 35 to $45 \mathrm{~Hz}$.

In literature, there are several different approaches to the analysis and comparison of EEG signals in participants suffering from different types of disorders and with different professions. Bhattacharya [11] gives a description of phase synchrony analysis of EEG. Differences in two groups, musicians and non-musicians were compared. An increase in phase synchrony, in delta and, more pronounced, in gamma frequency bands was observed in musicians, when the music listening task is compared to the resting phase or to the control condition. An increase in delta band was observed in non-musicians [11].

Differences between EEG signals of visual artists and non-artists were analyzed [12] using the power spectral density (PSD) during visual perception and mental imagery of paintings. The relative power values of the EEG signals were calculated [12]. The relative low beta $(15-18 \mathrm{~Hz})$ enhancement in Fp1, was proposed as the neurofeedback protocol for visual perception and mental imagery improvement of novice artists [12]. The difference between chess experts and novices was found in the delta frequency band, during resting time [13]. In the case of expert golfers, an increase in the right-hemisphere alpha wave activity is related to decreased errors [14]. There are numerous examples in literature applied in patients with psychological disorders. The relative power analysis of EEG signals has been conducted for participants with medically diagnosed autism [15]. The increasing value of the relative power in the gamma frequency band was shown during 18 sessions of neurofeedback protocol.

Commonly used methods and achievements of EEG diagnosis of ADHD were analyzed [16, 17]. Thetabeta power ratio (TBR) is a commonly used index for distinguishing between ADHD and non-ADHD, but unique and widely confirmed measure has not been determined so far. The increased value of the power TBR is thought to be indicative of a subgroup of ADHD [18] and less frequently used for classification of participants with ADHD compared to control group (non-ADHD) [19, 20]. In these papers, a measure was proposed stating that the mean TBR value of ADHD group exceeds 1.5 times standard deviation of mean TBR value for nonADHD. The accuracy of diagnosis using this measure was $84 \%$. EEG signals were recorded for 96 ADHD and 33 non-ADHD participants, with recording time of $90 \mathrm{sec}$ [20]. The measuring was done on $\mathrm{Cz}$ electrode. The recordings of EEG signals in [21] were made on 97 ADHD and 62 non-ADHD participants. The differences in TBR had an $89 \%$ accuracy in assessment. EEG signals were recorded on $\mathrm{Cz}$ electrode and the recording time was $10 \mathrm{~min}$. In the above-mentioned studies, the TBR measure had an excellent accuracy, but in the following studies it was not the case. In [22], EEG signals of 54 non-ADHD and 164 ADHD participants were studied, examined with the recording time of $<10$ minutes and the accuracy of diagnosis of the observed group was not calculated. The significant difference was seen in [22], in mean values and standard deviations of betatheta ratio (BTR). Fp1 and $\mathrm{Cz}$ electrodes were observed in the research procedure. In [23], the study was conducted by using signals with 2 min recording time in 169 ADHD and 167 non-ADHD participants. In this research, electrodes of significance were $\mathrm{Fz}$ and FCz. Same as in the previously mentioned research, the accuracy wasn't given, but the mean value of TBR in ADHD participants was 38\% higher than in non-ADHD. In [24], the absolute value of magnitude, the power value in theta and beta bands and their ratio were compared on $\mathrm{Cz}$ electrode with $63 \%$ accuracy of diagnostic using TBR differences (62 ADHD and 39 non-ADHD). Considering signals of 370 ADHD and 100 non-ADHD participants, in [25], the difference in TBR on $\mathrm{Cz}$ electrode was compared, and the obtained accuracy was only $38 \%$ (recording time was $5 \mathrm{~min}$ ). In comparison, when measuring TBR signals on CZ in [26] and [27], the 
accuracies of diagnostic were 53\% (54 ADHD and 51 non-ADHD) and 49\% (62 ADHD and 55 nonADHD). In [28] many more electrodes were used for diagnosed disorder (9 frontal electrodes), and the differences in relative power of theta and beta bands, alpha-beta ratio and theta-alpha ratio were compared (7 ADHD and 3 non-ADHD, with a diagnose accuracy of 97\%). In [29], the EEG signals of 80 ADHD and 59 healthy children were observed and power spectrum of each frequency band was calculated. One conclusion was that the power of beta band was significantly higher in ADHD participants [29].

In the treatment of ADHD most frequently used neurofeedback protocols are TBR, slow cortical potentials (SCP) and sensorimotor rhythm protocols [31, 32]. Neurofeedback trainings use skill learning principle to enable participants to acquire how to control their EEG and as a consequence of that change their brain state and activity to be approximate to a typically developing child [33]. A large number of participants is needed to analyze the effect of neurofeedback therapy [34].

Based on the results of previous studies, we decided to observe the mean and grand-mean values of magnitude and power theta-high beta ratios (THBR), but also the theta-alpha (TAR), theta-low beta (TLBR), alpha-delta (ADR) and delta-alpha (DAR) ratios on all epochs. In this study we decided to increase the number of electrodes and observe signals that were recorded on the F3, Fz, F4, C3, Cz, C4, P3, $\mathrm{Pz}$ and $\mathrm{P} 4$ electrodes, all shown in Figure 1 encircled in red.

\section{Experimental investigation}

\subsection{EEG data signal processing}

We used EEG raw data signal of people with ADHD and without ADHD. The recording conditions were opened eyes, closed eyes, hyperventilation, posthyperventilation, body moving.

Figure 2 shows a block-diagram of data signal processing procedure. Raw data signals were normalized and DC component of observed signal was removed. The frequency range of interest is between 1 and $47 \mathrm{~Hz}$. In the next step artifacts were removed. In neurofeedback, commonly used methods for artifacts use simple algorithms. We decided to remove artifacts in the following way: when the algorithm detects crossing over the maximum allowed value or minimum allowed value, the algorithm is searching backward the first zero

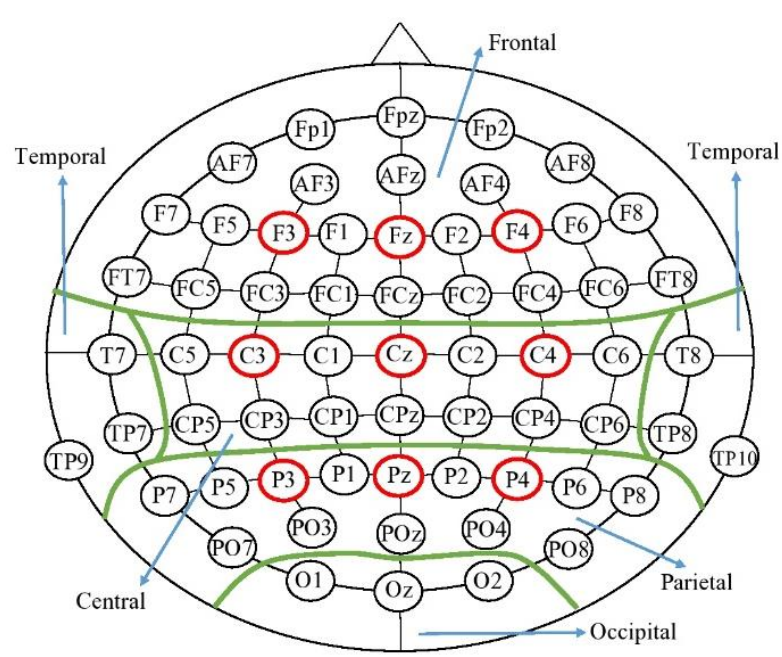

Figure 1. Placement of electrodes

crossing and forward the second zero crossing. Then the segment of the signal with values higher than the maximum allowable value or lower than the minimum allowable value is removed from further analysis. Figure 3 shows the visual description of artifacts' removal procedure. The procedure explained in this section was used for this preliminary study. We are planning to use a more complex way of artifacts removal in the future. The next step was a spectrum evaluation by using Fast Fourier Transform (FFT) [30]. The spectrum obtained by the FFT was divided into six observed frequency ranges $\left(\right.$ delta $\left(\delta_{\mathrm{f}}\right)$

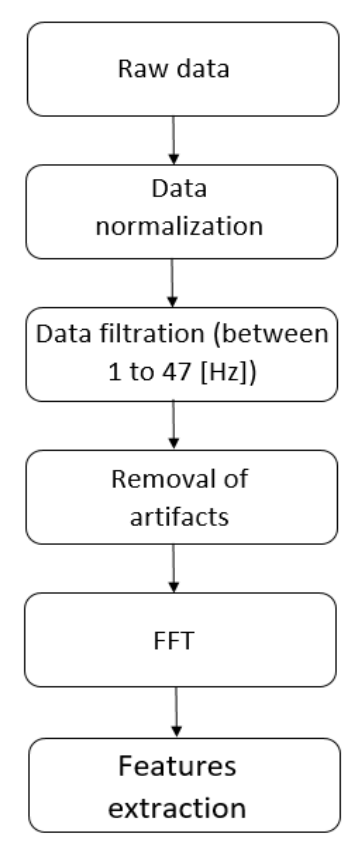

Figure 2. Block-diagram of observed data signal processing 

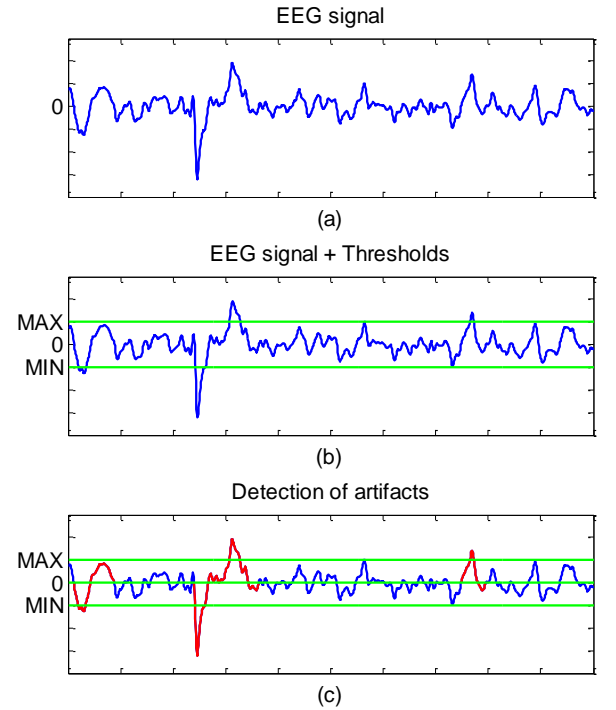

Signal after the removal of artifacts

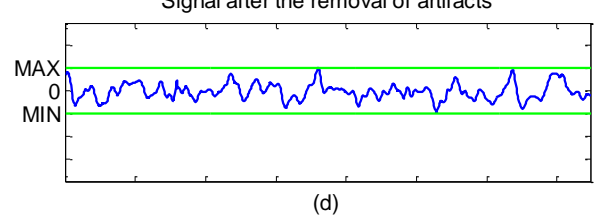

Figure 3. Removal of artifacts

$1-4 \mathrm{~Hz}$, theta $\left(\theta_{\mathrm{f}}\right) 4-8 \mathrm{~Hz}$, alpha $\left(\alpha_{\mathrm{f}}\right) 8-13 \mathrm{~Hz}$, low beta $\left(\beta_{\mathrm{Lf}}\right) 13-18 \mathrm{~Hz}$, high beta $\left(\beta_{\mathrm{Hf}}\right) 18-30 \mathrm{~Hz}$, gamma $\left.\left(\gamma_{\mathrm{f}}\right) 35-45 \mathrm{~Hz}\right)$. For the purpose of this paper, we have divided the beta frequency range into low beta and high beta because of more detailed signal comparison within the beta frequency range. We used raw data signals of ADHD and non-ADHD participants with sampling frequency of $256 \mathrm{~Hz}$. FFT was repeatedly applied on non-overlapping windows of $1 \mathrm{~s}$ duration. The result is the spectrum analysis with a frequency resolution of $1 \mathrm{~Hz}$. Data were further averaged to obtain the mean value for $15 \mathrm{~s}$ epochs as usually done in neurofeedback protocols.

\subsection{Features}

Features (power ratios, mean power value, grand mean power value, standard deviation, variance) were extracted and comparisons were made for two groups of subjects (ADHD and non-ADHD).

The relative power value of each frequency band in one epoch was calculated with the formula:

$$
P_{\Delta \delta}=\frac{\sum_{\Delta \delta_{\text {band }}}\left(\left|f_{i}\right|\right)^{2}}{\sum_{\text {all }}\left(\left|f_{i}\right|\right)^{2}},
$$

where $P_{\Delta \delta}$ is relative power of delta $(\delta)$ band per one epoch; $f_{i}$ are FFT coefficients at i-th spectral component. The formula described above is also valid for other frequency bands $\left(\delta_{f}, \theta_{f}, \alpha_{f}, \beta_{L f}, \beta_{H f}, \gamma_{f}\right)$.

\section{Results and discussion}

\subsection{Comparing the obtained features}

The results of previous studies indicate that $30-40 \%$ of ADHD is thought to have an increased value of TBR as pattern of activity [17, 18, 35, 36]. Other studies mentioned in introduction indicate that an increased value of TBR can be used as an indicator of ADHD in comparison with non-ADHD.

We included seventeen participants, three with medically diagnosed ADHD and fourteen without the diagnosis.

The same processing procedure to detect and remove artifacts and extract TBR values was used for all participants.

In this paper, we have tried to confirm the assumption that ADHD group has higher TBR when compared to non-ADHD group of participants, which was shown in the previous researches. In addition, we have analyzed other ratios and new indicators for diagnosis of ADHD disorder have been found.

Figures 4 and 5 show grand-mean values (GM) of magnitude spectral distributions in ADHD and nonADHD groups through all observing electrodes.

There is no difference in GM magnitude values of alpha and delta bands on electrodes F3, Fz and F4.

The same observation is confirmed for $\mathrm{GM}$ on $\mathrm{C} 3, \mathrm{Cz}$ and $\mathrm{C} 4$. However, there is bigger difference on electrodes $\mathrm{P} 3, \mathrm{Pz}$ and $\mathrm{P} 4$ for the ADHD group in alpha and delta bands as opposed to non-ADHD. Table 1 shows mean values and GM values of TLBR and ADR on the $\mathrm{Cz}$. The GM values TLBR and ADR on electrode $\mathrm{Cz}$ are $20.15 \%$ and $122.99 \%$ higher for the ADHD group in comparison with non-ADHD. Based on the percentage of difference in GM values which are calculated for both groups of participants and all epochs we can conclude that the main differences are seen in ADR.

Figure 6 shows GM values of TLBR and ADR on C3, $\mathrm{Cz}, \mathrm{C} 4, \mathrm{P} 3, \mathrm{Pz}$ and $\mathrm{P} 4$ electrodes. The difference of TLBR between observing groups was confirmed only on $\mathrm{Cz}$ [20] and $\mathrm{P} 4$ electrodes in comparison with previous researches. 

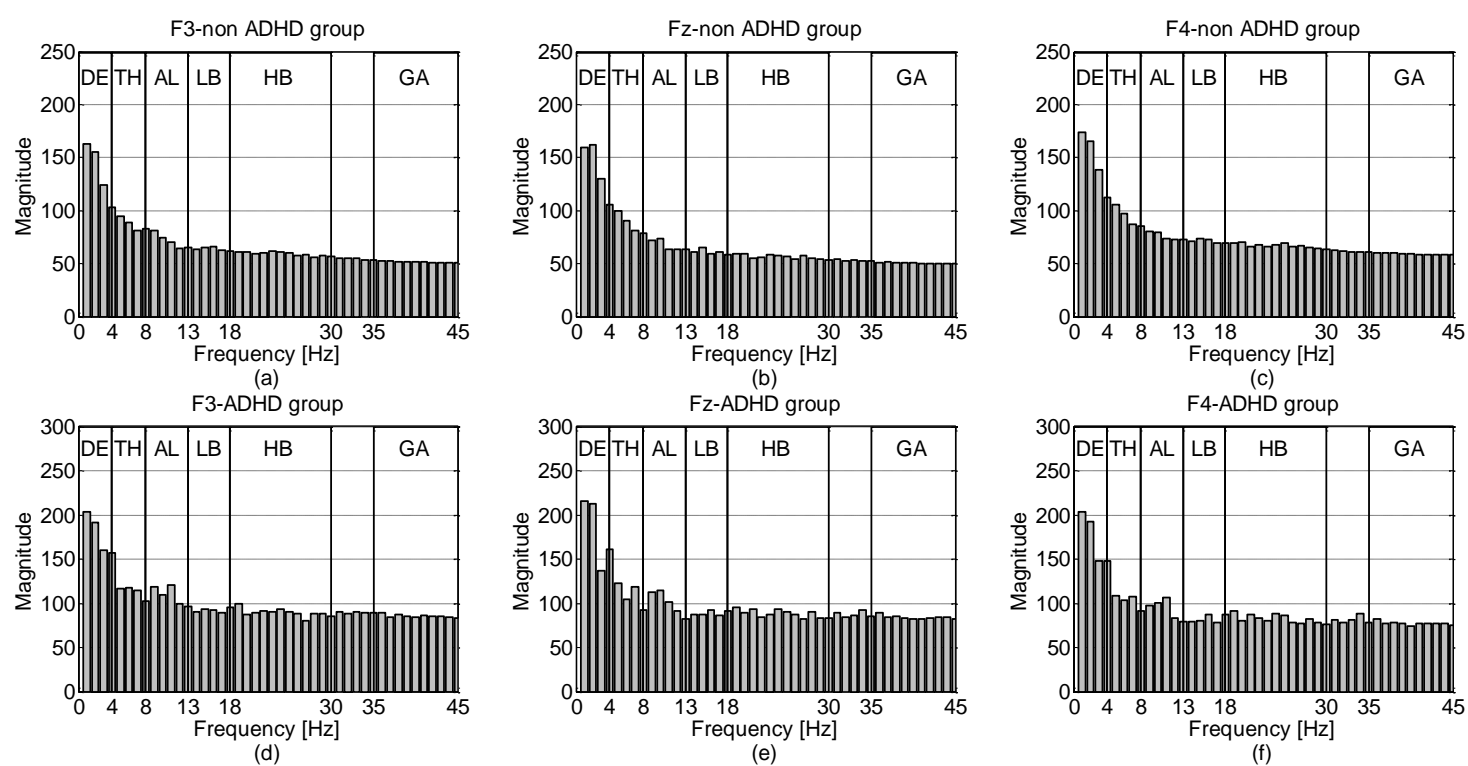

Figure 4. Grand-mean values of magnitude spectral distributions on F3, Fz and F4 electodes)
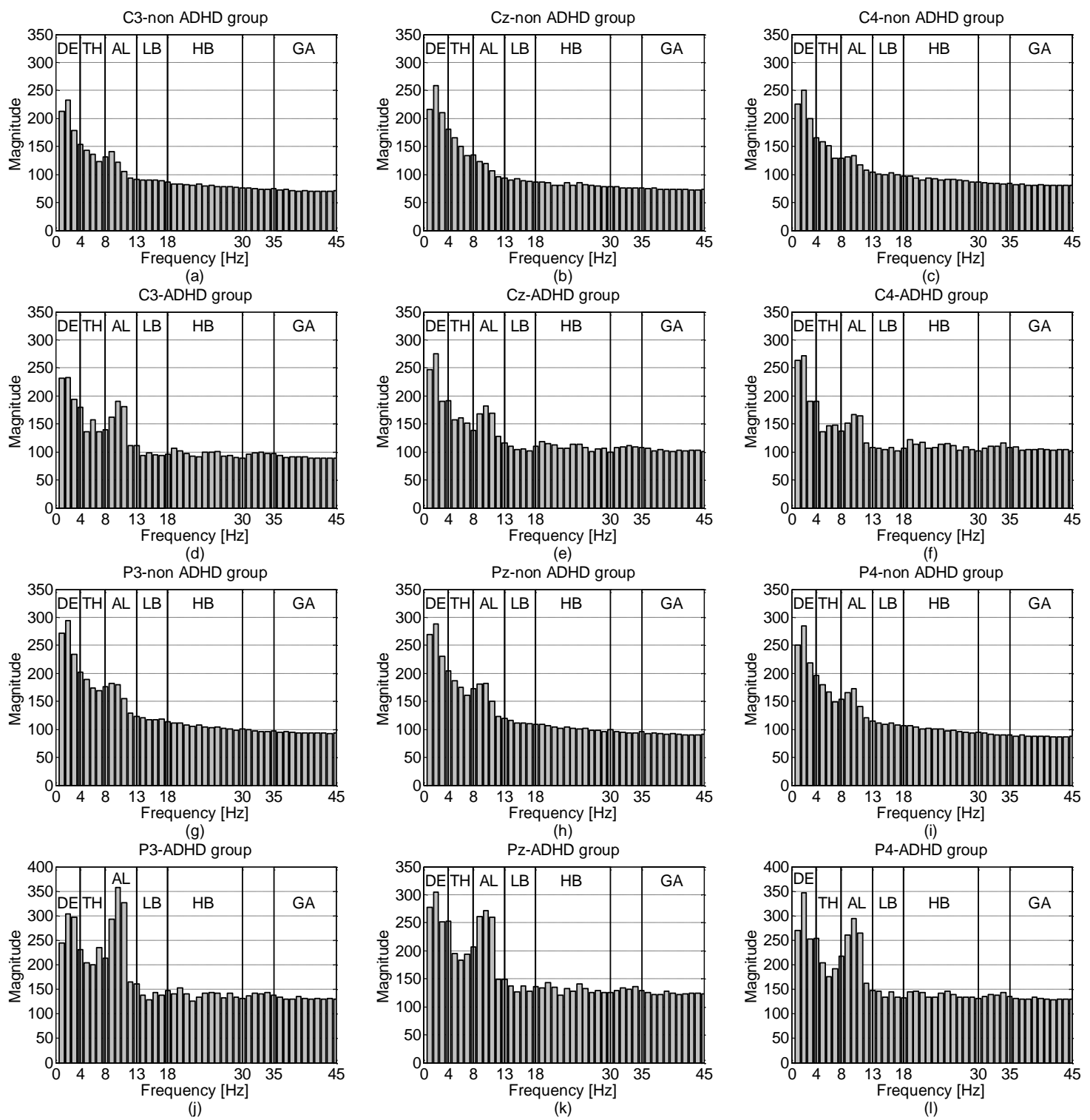

Figure 5. Grand-mean values of magnitude spectral distributions on $\mathrm{C3}, \mathrm{Cz}, \mathrm{C4}, \mathrm{P3}, \mathrm{Pz}$ and $\mathrm{P} 4$ electrodes, for $A D H D$ and non-ADHD participants (DE - delta, TH - theta, $A L$ - alpha, LB - low-beta, $H B$ high-beta, GA - gamma) 
Table 1. Mean values and grand-mean values of power TLBR and ADR on $C z$ (N-number)

\begin{tabular}{|l|c|c|c|}
\hline Group & $\mathrm{N}$ & TLBR & ADR \\
\hline ADHD & 1 & 4.75778 & 1.31432 \\
\cline { 2 - 4 } & 2 & 9.96931 & 0.50149 \\
\cline { 2 - 4 } & 3 & 2.15027 & 1.57408 \\
\hline GM ADHD & & 5.62579 & 1.12996 \\
\hline non-ADHD & 1 & 5.56906 & 0.541204 \\
\cline { 2 - 4 } & 2 & 6.67911 & 0.548517 \\
\cline { 2 - 4 } & 3 & 2.48677 & 0.677761 \\
\cline { 2 - 4 } & 4 & 2.13537 & 0.683455 \\
\cline { 2 - 4 } & 5 & 4.75630 & 0.302550 \\
\cline { 2 - 4 } & 6 & 2.84018 & 0.296510 \\
\cline { 2 - 4 } & 7 & 2.16688 & 0.910122 \\
\cline { 2 - 4 } & 8 & 7.54455 & 0.255433 \\
\cline { 2 - 4 } & 9 & 3.65058 & 0.765094 \\
\cline { 2 - 4 } & 10 & 9.76422 & 0.659966 \\
\cline { 2 - 4 } & 11 & 4.75630 & 0.302550 \\
\cline { 2 - 4 } & 12 & 6.71936 & 0.428446 \\
\cline { 2 - 4 } & 13 & 2.83285 & 0.472008 \\
\hline & 14 & 3.65279 & 0.250629 \\
\hline GM non-ADHD & 4.68245 & 0.50673 \\
\hline
\end{tabular}
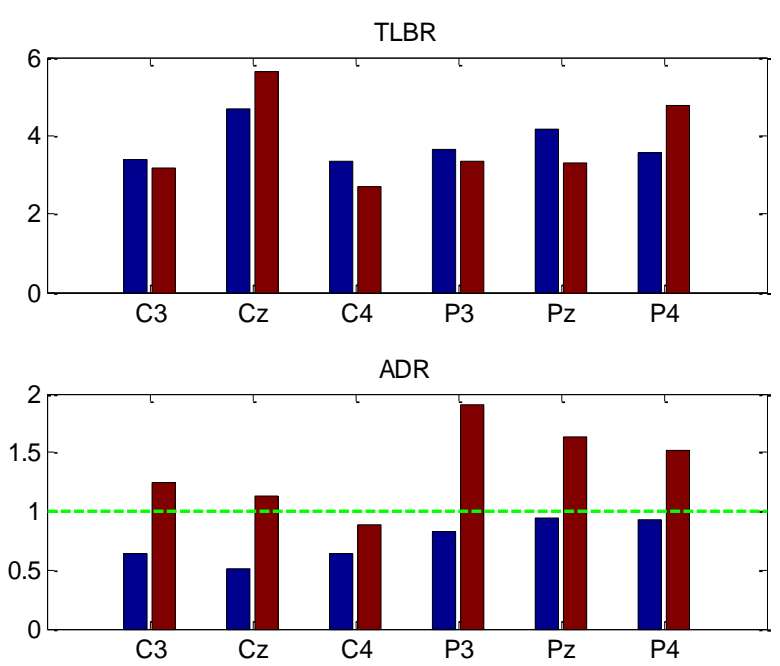

Figure 6. Grand-mean values of power TLBR and $A D R$ on $C 3, C z, C 4, P 3, P z$ and $P 4$ electrodes (blue - non-ADHD, red ADHD)
Table 2. Mean values and grand-mean values of power $\mathrm{ADR}$ on $\mathrm{C} 3, \mathrm{Cz}, \mathrm{C4}, \mathrm{P3}, \mathrm{Pz}$ and $\mathrm{P} 4$ (N-number, GM-grand mean)

\begin{tabular}{|c|c|c|c|c|c|c|}
\hline & $\mathrm{C} 3$ & $\mathrm{Cz}$ & $\mathrm{C} 4$ & $\mathrm{P} 3$ & $\mathrm{Pz}$ & $\mathrm{P} 4$ \\
\hline \multicolumn{7}{|c|}{ ADHD } \\
\hline 1 & 1.02 & 1.31 & 1.18 & 2.37 & 3.02 & 2.01 \\
\hline 2 & 0.50 & 0.50 & 0.40 & 1.13 & 0.51 & 0.67 \\
\hline 3 & 2.21 & 1.57 & 1.08 & 2.23 & 1.36 & 1.87 \\
\hline GM & 1.24 & 1.13 & 0.89 & 1.91 & 1.63 & 1.52 \\
\hline \multicolumn{7}{|c|}{ non-ADHD } \\
\hline 1 & 0.67 & 0.54 & 0.99 & 1.08 & 1.91 & 1.51 \\
\hline 2 & 0.61 & 0.55 & 0.71 & 0.85 & 1.31 & 1.55 \\
\hline 3 & 0.73 & 0.68 & 0.73 & 1.16 & 1.07 & 0.99 \\
\hline 4 & 0.78 & 0.68 & 0.68 & 0.69 & 0.65 & 0.72 \\
\hline 5 & 0.53 & 0.30 & 0.46 & 0.45 & 0.38 & 0.33 \\
\hline 6 & 0.36 & 0.29 & 0.43 & 0.59 & 0.55 & 0.62 \\
\hline 7 & 1.35 & 0.91 & 1.23 & 1.86 & 1.69 & 1.27 \\
\hline 8 & 0.42 & 0.26 & 0.38 & 0.25 & 0.26 & 0.32 \\
\hline 9 & 0.89 & 0.77 & 0.87 & 0.90 & 1.23 & 1.05 \\
\hline 10 & 0.75 & 0.66 & 0.68 & 0.92 & 0.83 & 0.86 \\
\hline 11 & 0.53 & 0.30 & 0.46 & 0.45 & 0.38 & 0.33 \\
\hline 12 & 0.44 & 0.43 & 0.37 & 0.48 & 0.46 & 0.39 \\
\hline 13 & 0.45 & 0.47 & 0.61 & 1.41 & 2.06 & 2.72 \\
\hline 14 & 0.43 & 0.25 & 0.34 & 0.43 & 0.35 & 0.33 \\
\hline GM & 0.64 & 0.51 & 0.64 & 0.82 & 0.94 & 0.93 \\
\hline
\end{tabular}

Table 2 shows that GM values of power ADR of ADHD participants are higher than 1 on all observing electrodes (except on $\mathrm{C} 4$ electrode) compared with non-ADHD participants whose values are lower than 1. Even if C4 electrode GM value of power ADR is lower than 1, it was higher for ADHD in comparison with non-ADHD subjects. Comparison of GM values for TLBR and ADR are presented in Figure 6.

\section{Conclusion}

In this article, a study was conducted to investigate differences between the EEG signals of participants with and without medically diagnosed ADHD. In addition, we have given a brief overview of previous studies on this topic. Given the results of this study, it can be mentioned that the increase in grand mean values of power TLBR on a $\mathrm{Cz}$ electrode was confirmed when comparing ADHD participants and 
non-ADHD participants, which was mentioned as conclusion in previous studies. ADR on $\mathrm{C} 3, \mathrm{Cz}, \mathrm{P} 3$, $\mathrm{Pz}$ and $\mathrm{P} 4$ is consistently higher for ADHD subjects. We propose that the ADR parameter should also be included in diagnostic procedures, but this needs to be verified or revised because of the small number of participants and lack of statistical analysis caused by a small number of participants.

\section{References}

[1] Sroubek, A., Kelly, M., Li, X.: Inattentiveness in attention - deficit / hyperactivity disorder, Neuroscience Bulletin, 29 (2013), 1, 103-110.

[2] Clauss-Ehlers C. S.: Encyclopedia of CrossCultural School Psychology, Springer Science \& Business Media, New York, 2010.

[3] National Institute of Mental Health, Attention Deficit Hyperactivity Disorder [Online], Available:

https://www.nimh.nih.gov/health/topics/attenti on-deficit-hyperactivity-disorder-

adhd/index.shtml\#part_145444. [Accessed: July 23, 2016].

[4] Diagnostic and Statistical Manual of Mental Disorders, Fifth Edition, Fifth Edit, American Psychiatric Association, 2013.

[5] Vos, T., Allen, C., et al.: Global, regional, and national incidence, prevalence, and years lived with disability for 310 diseases and injuries, 1990-2015: a systematic analysis for the Global Burden of Disease Study 2015, The Lancet, 388 (2016), 10053, 1545-1602.

[6] Napoletano, M.: Neurofeedback: Frequently Asked Questions [Online], Available: http://www.childandfamilycenter.com/services -specialized-neurofeedback.htm [Accessed: April 19, 2016].

[7] Electroencephalography [Online], Available: https://en.wikipedia.org/wiki/Electroencephalo graphy [Accessed: September 20, 2018].

[8] Sanei, S., Chambers, J.A.: EEG Signal Processing, John Wiley \& Sons Ltd, 2007.

[9] Marzbani, H., Marateb, H.R., Mansourian, M.: Methodological note: Neurofeedback: A comprehensive review on system design, methodology and clinical applications, Basic and Clinical Neuroscience, 7 (2016), 2, 143158.

[10] Jena, S.: Examination stress and its effect on $E E G$, International Journal of Medical Science and Public Health, 4 (2015), 11, 1493-1497.

[11] Bhattacharya, J., Petsche, H.: Phase synchrony analysis of EEG during music perception reveals changes in functional connectivity due to musical expertise, Signal Processing, 85 (2005), 11, 2161-2177.

[12] Shourie, N., Firoozabadi, M., Badie, K.: Neurofeedback training protocols based on spectral EEG feature subset and channel selection for performance enhancement of novice visual artists, Biomedical Signal Processing and Control, 43 (2018), 117-129.

[13] Volke, H.J., Dettmar, P., et al.: On-Coupling and Off-Coupling of Neocortical Areas in Chess Experts and Novices, Journal of Psychophysiology, 16 (2002), 1, 23-36.

[14] Crews, D.J., Landers, D.M.: Electroencephalographic measures of attentional patterns prior to the golf putt, Medicine \& Science in Sports \& Exercise, 25 (1993), 1, 116-126.

[15] Wang, Y., Sokhadze, E.M., et al.: Relative Power of Specific EEG Bands and Their Ratios during Neurofeedback Training in Children with Autism Spectrum Disorder, Frontiers in Human Neuroscience, 9 (2016), January,

[16] Lenartowicz, A., Sandra K. Loo: Use of EEG to Diagnose ADHD, Curr Psychiatry Rep., 16 (2015), 11,

[17] Arns, M., Conners, C.K., Kraemer, H.C.: A Decade of EEG Theta/Beta Ratio Research in ADHD: A Meta-Analysis, Journal of Attention Disorders, 17 (2013), 5, 374-383.

[18] Bussalb, A., Collin, S., et al.: Is there a cluster of high theta-beta ratio patients in attention deficit hyperactivity disorder?, Clinical Neurophysiology, 130 (2019), 8, 1387-1396.

[19] Monastra, V.J., Fenger, T.N., et al.: Assessing attention deficit hyperactivity disorder via quantitative electroencephalography: An initial validation study., Neuropsychology, 13 (1999), 3, 424-433.

[20] Monastra, V.J., Lubar, J.F., Linden, M.: The development of a quantitative electroencephalographic scanning process for attention deficit-hyperactivity disorder: Reliability and validity studies, Neuropsychology, 15 (2001), 1, 136-144.

[21] Snyder, S.M., Quintana, H., et al.: Blinded, multi-center validation of EEG and rating scales in identifying ADHD within a clinical sample, Psychiatry Research, 159 (2008), 3, 346-358.

[22] González-Castro, P., Álvare, L., et al.: Cortical activation and attentional control in ADAH 
subtypes, International Journal of Clinical and Health Psychology, 10 (2010), 1, 23-39.

[23] Williams, L.M., Hermens, D.F., et al.: Using Brain-Based Cognitive Measures to Support Clinical Decisions in ADHD, Pediatric Neurology, 42 (2010), 2, 118-126.

[24] Ogrim, G., Kropotov, J., Hestad, K.: The quantitative EEG theta/beta ratio in attention deficit/hyperactivity disorder and normal controls: Sensitivity, specificity, and behavioral correlates, Psychiatry Research, 198 (2012), 3, 482-488.

[25] Loo, S.K., Cho, A., et al.: Characterization of the Theta to Beta Ratio in ADHD: Identifying Potential Sources of Heterogeneity, Journal of Attention Disorders, 17 (2013), 5, 384-392.

[26] Liechti, M.D., Valko, L., et al.: Diagnostic value of resting electroencephalogram in attentiondeficit/ hyperactivity disorder across the lifespan, Brain Topography, 26 (2013), 1, 135151.

[27] Buyck, I., Wiersema, J.R.: Resting electroencephalogram in attention deficit hyperactivity disorder: Developmental course and diagnostic value, Psychiatry Research, 216 (2014), 3, 391-397.

[28] Abibullaev, B., An, J.: Decision support algorithm for diagnosis of ADHD using electroencephalograms, Journal of Medical Systems, 36 (2012), 4, 2675-2688.

[29] Kamida, A., Shimabayashi, K., et al.: EEG power spectrum analysis in children with $A D H D$, Yonago Acta Medica, 59 (2016), 2,
169-173.

[30] Cooley, J.W., Tukey, J.W.: An Algorithm for the Machine Calculation of Complex Fourier Series, Mathematics of Computation, 19 (2006), 90, 297.

[31] Arns, M., Heinrich, H., Strehl, U.: Evaluation of neurofeedback in ADHD: The long and winding road, Biological Psychology, 95 (2014), 1, 108115.

[32] Mayer, K., Blume, F., et al.: Neurofeedback of slow cortical potentials as a treatment for adults with Attention Deficit-/Hyperactivity Disorder, Clinical Neurophysiology, 127 (2016), 2, 13741386.

[33] Van Doren, J., Arns, M., et al.: Sustained effects of neurofeedback in ADHD: a systematic review and meta-analysis, European Child and Adolescent Psychiatry, 28 (2019), 3, 293-305.

[34] Zolubak, M., Pelc, M., Kawala-Janik, A.: Challenges in differentiating between attention disorders based on EEG recordings in neurofeedback therapy, 2018 Applications of Electromagnetics in Modern Techniques and Medicine, PTZE 2018, (2018), 65-68.

[35] Zhang, D.W., Roodenrys, S., et al.: Atypical interference control in children with $A D / H D$ with elevated theta/beta ratio, Biological Psychology, 128 (2017), April, 82-88.

[36] Clarke, A.R., Barry, R.J., et al.: EEG-defined subtypes of children with attentiondeficit/hyperactivity disorder, Clinical Neurophysiology, 112 (2001), 11, 2098-2105. 